\title{
Review: lower dose combination antihypertensive therapy is preferable to standard dose single drug therapy
}

Law MR, Wald NJ, Morris JK, Jordan RE. Value of low dose combination treatment with blood pressure lowering drugs: analysis of 354 randomised trials. BMJ 2003;326:1427-34.

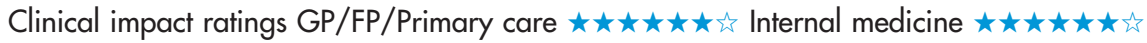

What is the safety and efficacy of different doses and combinations of 5 main categories of blood pressure (BP) lowering drugs?

\section{METHODS}

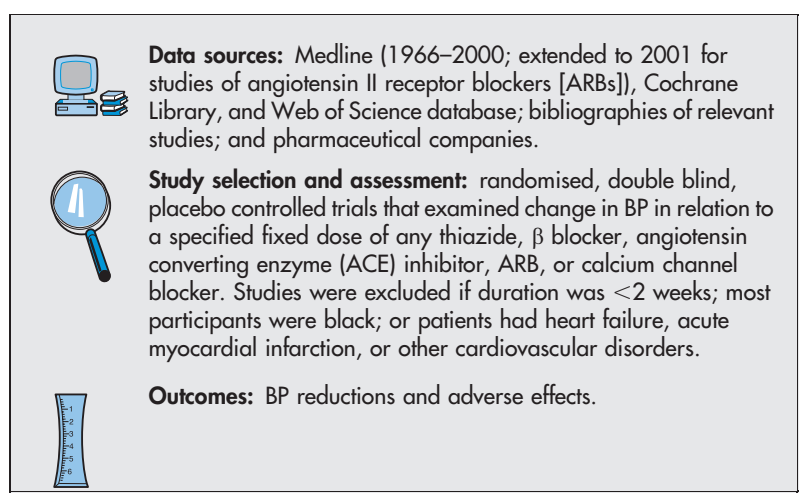

\section{MAIN RESULTS}

354 trials (median duration $4 \mathrm{wk}$ ) met the selection criteria. The trials included 791 treatment groups (219 in crossover and 572 in parallel group trials) that tested different drugs or different doses of the same drug. 7 thiazides, $15 \beta$ blockers, 12 ACE inhibitors, 8 ARBs, and 11 calcium channel blockers were studied. 39879 participants (mean age 53 y) received treatment and 15817 participants (mean age 53 y) received placebo.

Data were synthesised by meta-analysis. The 5 drug categories gave similar BP reductions. The mean placebo adjusted reduction was 9.1 $\mathrm{mm} \mathrm{Hg} \mathrm{(95 \%} \mathrm{CI} 8.8$ to 9.3 ) systolic and $5.5 \mathrm{~mm} \mathrm{Hg}$ (CI 5.4 to 5.7 ) diastolic at standard doses ("usual maintenance doses"), and $7.1 \mathrm{~mm}$ Hg (CI 6.8 to 7.5 ) systolic and $4.4 \mathrm{~mm} \mathrm{Hg} \mathrm{(CI} 4.2$ to 4.6 ) diastolic at half standard doses. The different drugs within each of the 5 categories gave similar BP reductions. For every $10 \mathrm{~mm} \mathrm{Hg}$ increase in pretreatment $\mathrm{BP}$, the reduction in $\mathrm{BP}$ with $\mathrm{I}$ drug at standard dose increased by a mean of $1.0 \mathrm{~mm} \mathrm{Hg}$ (CI 0.7 to 1.2 ) systolic and $1.1 \mathrm{~mm}$ $\mathrm{Hg}$ (CI 0.8 to 1.4 ) diastolic.

50 trials (119 placebo controlled comparisons) tested the effect of drugs of 2 categories separately and in combination. The BP lowering effects of different drug categories were additive. 2 drugs used in combination gave mean placebo adjusted reductions in BP by 14.6 $\mathrm{mm} \mathrm{Hg}$ systolic and $8.6 \mathrm{~mm} \mathrm{Hg}$ diastolic.

For correspondence: Professor $\mathrm{M} \mathrm{R}$ Law, University of London, London, UK. m.r.law@qmul.ac.uk

Source of funding: no external funding.
Adverse effects of thiazides, $\beta$ blockers, and calcium channel blockers were dose related. The main adverse effect with ACE inhibitors was cough, and this did not vary with dose. ARBs were not associated with excess of adverse effects. In 66 trial arms, single drugs caused adverse effects in $5.2 \%$ (CI 3.6 to 6.6) of participants; and, in 33 trial arms, 2 drugs used in combination caused adverse effects in $7.5 \%$ (5.8\% to $9.3 \%)$ of participants, which was less than additive.

\section{CONCLUSIONS}

The 5 main categories of blood pressure (BP) lowering drugs give similar reductions in BP. Within each drug category, individual drugs give similar BP reductions. When drugs are used in combination, the BP reductions are additive, but the adverse effects are less than additive.

Abstract and commentary also appear in ACP Journal Club.

\section{Commentary}

The valuable and well researched review by Law et al investigates the effectiveness of the 5 most frequently used drugs for the pharmacological treatment of hypertension. This review is useful to guide practitioners in making empirically based prescription decisions that balance drug effectiveness with minimal side effects, to help policy makers in identifying similarly effective drugs so that maximum patient benefit can be achieved at the lowest cost (ie, facilitate reference based pricing), and to provide researchers with critical normative data against which to compare the effectiveness of new treatments.

The careful investigation and display of dose response curves and (partially) corresponding increases in side effects will be much appreciated by practitioners, and the huge patient base underlying these numbers speaks for the trustworthiness of the findings. All general and internal medicine practitioners should be familiar with the contents and conclusions of this review.

Although not a criticism of the work presented, the hypertension findings aggregated in the review treat the disease (and the patients presenting with high BP) as homogeneous. Individual patients still need to be carefully assessed for other risk factors and the presence of comorbid conditions (eg, age, diabetes, kidney dysfunction, depression, and nonadherence) so that treatment choices can be tailored to their individual circumstances. Unfortunately, this is exactly where evidence is most lacking.

Wolfgang Linden, PhD, RPsych University of British Columbia Vancouver, British Columbia, Canada 